\title{
The Last Nanometer - Hydration Structure of DNA and Solid Surfaces Probed by Ultra High Resolution AFM
}

\author{
Uri Sivan*
}

Technion - Israel Institute of Technology, Haifa, Israel

*E-mail phsivan@technion.ac.il

Recent advancements in atomic force microscopy facilitate atomic-resolution threedimensional mapping of hydration layers next to macromolecules and solid surfaces. These maps provide unprecedented information on the way water molecules organize and bind these objects. Since the hydration structure governs the energetics of solvation and interactions between objects immersed in solution, the new data are invaluable when trying to resolve fundamental questions such as identification of molecular binding sites and interaction mechanisms.

After a short presentation of our home-built microscope, characterized by sub $0.1 \AA$ noise level, the talk will focus on two representative studies. The first one will disclose our recent finding [1] that in solutions in contact with atmosphere, hydrophobic surfaces are generically coated with a dense layer of adsorbed gas molecules. This layer renders the hydrophobic interaction a certain universality, regardless of the underlying surface. The second study will present our recent success [2] in obtaining ultra high resolution images of DNA and 3d maps of its hydration structure (e.g., Fig. 1 below). This study shows that labile water molecules concentrate along the DNA grooves, in agreement with known position of DNA binding sites.
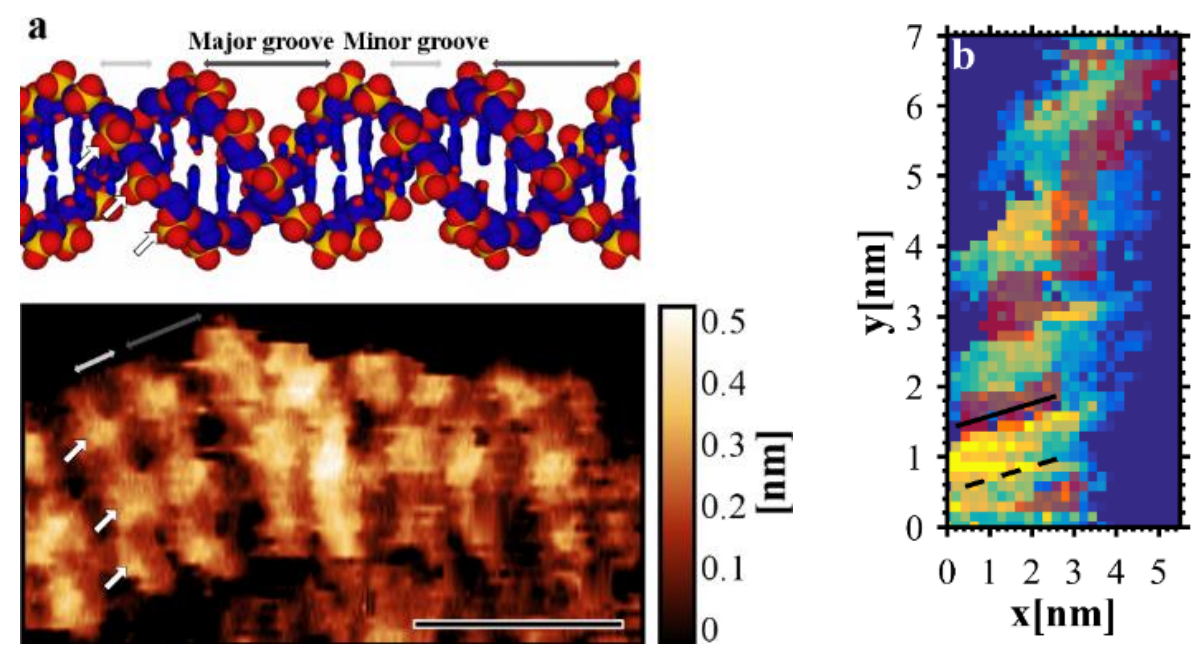

Figure 1: (a) An ultra high resolution image of DNA with a reference model of B-DNA. The major grooves, minor grooves and top-facing phosphates are highlighted with gray and white arrows on the model and the scan. Scale bar, $5 \mathrm{~nm}$. (b) Hydration of double stranded DNA. Red shaded pixels mark the position of labile water molecules.

\section{References}

[1] Itai Schlesinger and Uri Sivan, J. Am. Chem. Soc., 140, 10473-10481 (2018)

[2] Kfir Kuchuk and Uri Sivan, Nano Lett., 18, 2733-2737 (2018) 